\title{
RESULTADOS QUIRÚRGICOS DE LA HERNIOPLASTÍA INGUINAL LAPAROSCÓPICA CON TÉCNICA TRANSABDOMINAL PRE-PERITONEAL (TAPP)*
}

\author{
Dr. Mauricio Gabrielli N. ${ }^{1}$ \\ 1 Departamento de Cirugía Digestiva. Facultad de Medicina. Pontificia Universidad Católica de Chile. \\ Santiago, Chile.
}

\begin{abstract}
\section{Surgical outcomes of laparoscopic transabdominal pre-peritoneal inguinal hernia repair (TAPP)}

Introduction: Laparoscopic hernia repair was first described by Ger in 1982. Arregui et al. in 1992, reported the transabdominal preperitoneal procedure (TAPP). The debate of what is the best technique continues today. The aim of this study was to describe the technique and surgical outcomes including morbidity and recurrence after TAPP. Material and Methods: Retrospective cohort study. Data was obtained from the database of patients who underwent laparoscopic inguinal hernia repair with TAPP at Hospital Clínico Universidad Católica de Chile from October 2008 to December 2012. The follow-up was performed with postoperative controls and telephone calls, specifically asking for recurrence and pain. Results: Two-hundred and seventy-one TAPP procedures in 197 patients with inguinal hernia were included in the study. Twenty two patients had complications $(11.2 \%), 18$ of which corresponded to Clavien type I. Median follow-up was 18 months (12-28), including 94\% patients of this series. There were five recurrences, corresponding to $1.85 \%$ patients. Conclusions: In this study the outcomes of TAPP for inguinal hernia repair were similar to the outcomes reported in the current literature.

Key words: Inguinal hernia, preperitoneal, transabdominal.

\section{Resumen}

Introducción: La primera reparación herniaria laparoscópica fue descrita por Ger en 1982, Arregui y colaboradores en 1992 comunican el procedimiento de reparación transabdominal preperitoneal (TAPP). El debate sobre cuál es la mejor técnica aún persiste. El objetivo de este estudio es mostrar la técnica y los resultados quirúrgicos a corto plazo de la hernioplastía inguinal con la técnica TAPP. Material y Métodos: Estudio de cohorte retrospectiva. Los datos fueron obtenidos desde la base de datos de cirugía digestiva y registros clínicos de los pacientes a quienes se les realizó una hernioplastía inguinal por vía laparoscópica con técnica TAPP en el Hospital Clínico de la Pontificia Universidad Católica de Chile entre octubre de 2008 y diciembre

*Recibido el 23 de julio de 2014 y aceptado para publicación el 11 de septiembre de 2014.

El autor no refiere conflictos de interés.

Correspondencia: Dr. Mauricio Gabrielli N.

megabrielli@uc.cl 
de 2012. El seguimiento de los pacientes se realizó en los controles postoperatorios y vía telefónica, preguntando específicamente por recidiva y dolor crónico. Resultados: En el período de estudio fueron realizadas, en forma consecutiva 271 hernioplastías laparoscópicas en 197 pacientes con diagnóstico de hernia inguinal. Se registraron complicaciones postoperatorias en 22 pacientes $(11,2 \%)$, las que fueron de tipo I de Clavien en 18 pacientes. La mediana de seguimiento fue de 18 meses (12-28), alcanzando un 94\% de la serie. Durante el período de seguimiento se registró recidiva en 5 de las hernias operadas, lo que corresponde a un 1,85\%. Conclusiones: En este estudio los resultados de la técnica TAPP para la reparación de hernia inguinal demostraron ser similares a los publicados en la literatura.

Palabras clave: Hernia inguinal, transabdominal, preperitoneal.

\section{Introducción}

La hernioplastía inguinal es una de las operaciones más frecuentes del mundo ${ }^{1,2}$. Su historia es tan antigua que se remonta a registros de los médicos sumerios en Mesopotamia 4.000 años A.C., en donde aparece el concepto de "herniotomía"3,4. La era moderna de la cirugía de la hernia inguinal se inicia con el desarrollo de las técnicas que refuerzan las paredes del conducto inguinal, con resultados muy variables a excepción de lo mostrado con la técnica de Shouldice, en algunos centros 5 . Posteriormente surge el concepto "libre de tensión" con el uso de malla sintética, con mejores resultados que han sustentado su uso hasta el día de hoy ${ }^{6-8}$.

La primera reparación herniaria laparoscópica fue descrita por Ger en $1982^{9}$, en 1992 Arregui y colaboradores $^{10}$, comunican el procedimiento de reparación transabdominal preperitoneal (TAPP).

El debate sobre cuál es la mejor técnica aún persiste, muchos estudios aleatorizados han comparado las técnicas de Shouldice, Lichtenstein y reparación laparoscópica sin demostrar superioridad de una técnica sobre otra ${ }^{11,12}$.

La hernioplastía laparoscópica, según algunos autores, presenta beneficios por sobre las técnicas abiertas; tales como menor dolor post operatorio, menor estadía hospitalaria, reinserción laboral temprana y mejor calidad de vida ${ }^{11,12}$.

El objetivo de este estudio es mostrar la técnica y los resultados quirúrgicos a corto plazo de la hernioplastía inguinal vía TAPP.

\section{Material y Métodos}

\section{Diseño}

Estudio descriptivo de una cohorte retrospectiva. Los datos fueron obtenidos desde la base de datos de cirugía digestiva y registros clínicos de los pacientes en quienes se realizó una hernioplastía inguinal por vía laparoscópica con técnica TAPP en el Hospital Clínico de la Pontificia Universidad Católica de Chile entre octubre de 2008 y diciembre de 2012. Los resultados se agruparon en cuatro grupos; hombre hernia inguinal unilateral, hombre hernia inguinal bilateral, mujer hernia inguinal unilateral y mujer hernia inguinal bilateral.

\section{Selección de pacientes}

Se incluyeron pacientes mayores de 15 años, sin límite de edad superior, a quienes se les diagnosticó de forma clínica una hernia inguinal, o con el apoyo de una ecotomografía inguinal de partes blandas en caso de duda diagnóstica. La vía laparocópica se decidió para aquellos pacientes con hernias Tipo I, II y IIIA, de la clasificación de Nyhus ${ }^{13}$ (Tabla 1). En aquellos pacientes en que se diagnosticó una hernia inguinal contralateral durante la cirugía, ésta fue reparada en el mismo momento. Como criterios de exclusión se consideró aquellos pacientes menores de 15 años, pacientes con hernias recidivadas (Tipo IV de Nyhus) o hernias grandes Tipo IIIB o con el diagnóstico de hernia femoral. También se excluyeron aquellos pacientes con contraindicación de cirugía laparoscópica.

\section{Seguimiento}

El seguimiento fue realizado mediante controles clínicos post operatorios entre las dos primeras

Tabla 1. Clasificación de Nyhus para hernia inguinal

\begin{tabular}{|c|c|}
\hline Tipo I & $\begin{array}{l}\text { Indirecta, pequeña } \\
\text { Anillo interno normal } \\
\text { Saco en canal inguinal }\end{array}$ \\
\hline Tipo II & $\begin{array}{l}\text { Indirecta, mediana } \\
\text { Anillo interno alargado } \\
\text { Saco no en el escroto }\end{array}$ \\
\hline Tipo III & $\begin{array}{l}\text { A Directas pequeñas o medianas. } \\
\text { Sin protrusión por anillo interno } \\
\text { B Indirectas grandes Compromete la pared } \\
\text { posterior del conducto inguinal } \\
\text { C Hernia femoral }\end{array}$ \\
\hline Tipo IV & $\begin{array}{l}\text { Hernias recurrentes } \\
\text { A Indirectas } \\
\text { B Directas } \\
\text { C Femorales } \\
\text { D Combinación }\end{array}$ \\
\hline
\end{tabular}


semanas y 1 mes desde la intervención. Todos los pacientes tuvieron al menos un control médico en este período. Posteriormente se controlaron presencialmente aquellos pacientes que espontáneamente decidieron consultar por presentar alguna sintomatología en relación a la cirugía. Aquellos que no fueron controlados presencialmente fueron llamados telefónicamente, preguntándose explícitamente por dolor, recidiva o cualquier otra sintomatología. Se definió el seguimiento a corto plazo como aquel período comprendido entre los 30 días y 5 años desde la operación.

Las complicaciones postoperatorias fueron clasificadas de acuerdo a la Clasificación de Clavien, modificada por Kaafarani específicamente para patología herniaria ${ }^{14}$ (Tabla 2). Como dolor crónico se definió aquel que persistió luego de 1 año desde la cirugía.

\section{Técnica quirúrgica}

Paciente en posición supina con los brazos pegados al cuerpo. Vejiga vaciada en el preoperatorio inmediato. Monitor a los pies del paciente. Cirujano al lado opuesto de la hernia a intervenir. Neumoperitoneo con Veress a nivel umbilical, hasta $15 \mathrm{~mm}$ Hg. Primer trócar de $12 \mathrm{~mm}$ umbilical, dos trócares auxiliares de $5 \mathrm{~mm}$ en ambos flancos por fuera de la vaina de los rectos del abdomen. Uso de óptica de 0 grados. Trendelemburg de 15 grados aproximadamente. Visualización de ambas regiones inguinales.

Se comienza con la disección del peritoneo a nivel del arco aponeurótico del músculo transverso, con electrocoagulación, ya sea con tijera conectada al monopolar o gancho, desde la espina ilíaca anterosuperior hasta el ligamento umbilical medial, siempre sobre el ligamento inguinal profundo. En esta disección se debe incorporar el saco herniario.

Si se identifica una hernia directa, el saco herniario se reduce con facilidad, por otro lado, si es indirecta, éste se debe separar de los elementos del cordón espermático, siempre con disección roma. Posterior a ello se debe identificar el ligamento de Cooper; en algunas oportunidades este ligamento puede estar atravesado por un complejo de vasos conocidos como corona mortis. Estos vasos se pueden coagular de ser necesario.

Una vez completada la disección se procede a instalar la malla de tamaño adecuado para cubrir el defecto y todas las zonas potenciales de herniación futura. La malla se extiende desde la línea media hasta la espina ilíaca anterosuperior por encima del arco del músculo transverso. Para facilitar la postura de la malla se recomienda la tracción del borde superior del peritoneo aprovechando el neumoperitoneo que permite despegarlo. La malla se fija al Cooper $\mathrm{y}$ al arco aponeurótico de músculo transverso con
Tabla 2. Clasificación de Clavien para complicaciones en cirugía herniaria

\begin{tabular}{|c|c|}
\hline Tipo I & $\begin{array}{l}\text { Cualquier desviación del postoperatorio nor- } \\
\text { mal. Sin requerimiento de tratamiento farmaco- } \\
\text { lógico, endoscópico, radiológico o quirúrgico. } \\
\text { Aceptado el uso de analgésicos, antieméticos, } \\
\text { electrolitos, diuréticos antipiréticos }\end{array}$ \\
\hline Tipo II & $\begin{array}{l}\text { Requiere tratamiento farmacológico más allá } \\
\text { de lo indicado en la Tipo I. Como transfusiones } \\
\text { o nutrición parenteral total }\end{array}$ \\
\hline Tipo III & $\begin{array}{l}\text { Requiere cirugía, tratamiento endoscópico o } \\
\text { radiológico } \\
\text { A Intervención sin anestesia general } \\
\text { B Intervención bajo anestesia general }\end{array}$ \\
\hline Tipo IV & $\begin{array}{l}\text { Riesgo vital } \\
\text { A Disfunción de un órgano } \\
\text { B Disfunción multiorgánica }\end{array}$ \\
\hline Tipo V & Muerte \\
\hline
\end{tabular}

grapas. Cierre del peritoneo con engrapadora o sutura bajando la presión del neumoperitoneo a $8 \mathrm{~mm}$ Hg (Figura 1).

En todos los pacientes del estudio se realizó la misma técnica y se utilizó el mismo material protésico compuesto de polipropileno y monocryl de $10 \times 15 \mathrm{~cm}$.

\section{Análisis estadístico}

Las variables continuas se expresan como promedio y desviación estándar para datos paramétricos y en mediana y rango intercuartil (RIC) para datos no paramétricos (obtenido usando el test de ShapiroWilk). La comparación de medianas se realizó utilizando el test de Mann-Whitney. Las proporciones se compararon utilizando el test de $\chi^{2}$ o el test de Fisher (si alguno de los valores esperados en la tabla de contingencia es menor a 5). El análisis estadístico fue realizado utilizando el programa SPSS versión 20 .

\section{Resultados}

En el período de estudio fueron realizadas consecutivamente 271 hernioplastías laparoscópicas utilizando la técnica transabdominal pre-peritoneal (TAPP) en 197 pacientes con diagnóstico de hernia inguinal. En 72 pacientes (36,5\%) la hernia se presentó en el lado derecho, en 51 pacientes $(25,9 \%)$ en el lado izquierdo, mientras que en 74 pacientes $(37,6 \%)$ fue bilateral. El promedio de edad de la cohorte fue de $52 \pm 15,4$ años. Un $77,2 \%$ de los pacientes eran de sexo masculino. Un $57,9 \%$ de los 


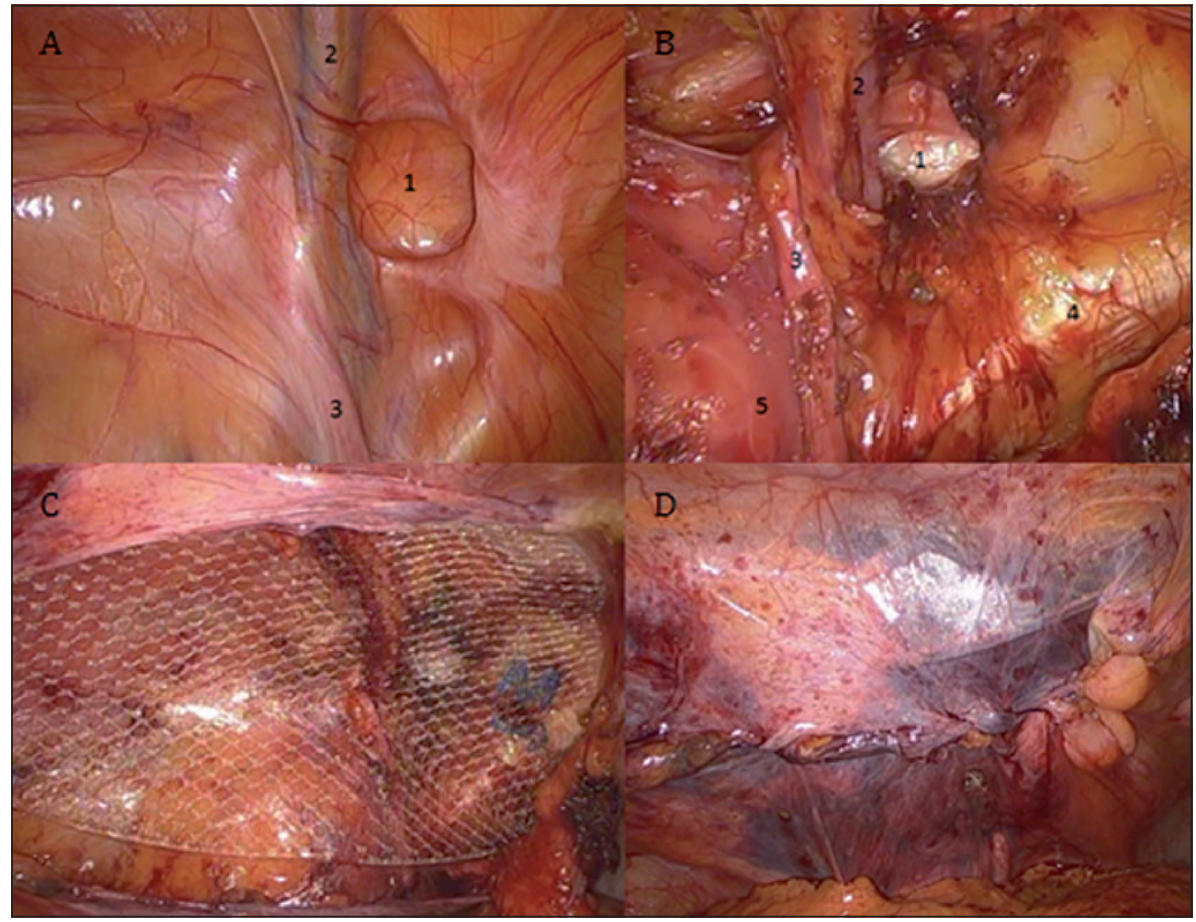

Figura 1. Hernioplastía inguinal izquierda laparoscópica en una paciente de sexo femenino. A. Visión intraperitoneal. 1) Hernia inguinal directa. 2) Vasos epigástricos. 3) Ligamento redondo. B. Visión preperitoneal. 1) Saco herniario reducido. 2) Vasos epigástricos. 3) Ligamento redondo. 4) Ligamento de Cooper. 5) Arteria ilíaca externa. C. Malla instalada. D. Visión del peritoneo suturado.

pacientes fueron clasificados como ASA I, un 40,6\% como ASA II y un $1,5 \%$ como ASA III. El resumen de las características preoperatorias se encuentra en la Tabla 3.

La mediana de evolución de síntomas derivados de la patología herniaria fue de 6 meses (3-12). En el caso de las hernias unilaterales, el diagnóstico se estableció de forma clínica en un 93,5\% de los pacientes y mediante una ecografía de partes blandas en un $6,5 \%$. En las hernias bilaterales, el diagnóstico fue clínico en un $87,8 \%$ de los pacientes y ecográfico en un 12,2\%. En los casos en que se diagnosticó de forma incidental intraoperatoria, una hernia contralateral, ésta se reparó en la misma intervención.

Con respecto al procedimiento quirúrgico, la mediana de tiempo operatorio en la cohorte completa fue de $60 \mathrm{~min}$ (58-90). Al comparar el tiempo operatorio entre procedimientos unilaterales y bilaterales, se obtuvo un mayor tiempo para los bilaterales (60 $\min (45-74)$ vs $90 \min (60-120), \mathrm{p}<0,001)$. Dentro de los procedimientos unilaterales, el tiempo quirúrgico fue mayor en el caso de los hombres (60 $\mathrm{min}$ (50-90) vs $45 \min (33-58), \mathrm{p}=0,002)$. En toda la cohorte estudiada no se presentaron complicaciones intraoperatorias, ni necesidad de conversión a ciru- gía clásica. La mediana de tiempo de hospitalización fue de 1 día (1-2). No hubo diferencias en el tiempo de hospitalización postoperatoria entre los pacientes operados por hernia unilateral y bilateral (1 día vs 1 día, $\mathrm{p}=0,88)$.

Se registraron complicaciones postoperatorias en 22 pacientes $(11,2 \%)$. Éstas fueron clasificadas como tipo I en 18 pacientes ( 9 hematomas, 5 seromas, 2 hidroceles, 2 retenciones urinarias; 9,2\%), tipo IIIa en 2 pacientes ( 2 seromas que requirieron punción; $1 \%$ ). Tipo IIIb en 1 paciente (epiplocele que requirió cirugía; $0,5 \%$ ) y tipo $\mathrm{V}$ en 1 paciente (infarto agudo al miocardio; $0,5 \%$ ). No se encontró diferencia estadísticamente significativa al comparar la aparición de complicaciones entre los pacientes operados por hernia unilateral y bilateral $(13 \%$ vs $9 \%, p=0,4)$.

La mediana de seguimiento fue de 18 meses (12-28), alcanzando a un $94 \%$ de la serie. Durante el período de seguimiento se registró recidiva en 5 $(1,85 \%)$ de las hernias operadas. No hubo diferencias en la recidiva de hernias unilaterales vs bilaterales $(2,4 \%$ vs $1,4 \%, p=0,8)$. No hubo diferencias al comparar la recidiva herniaria entre hombres y mujeres en el grupo total $(1,9 \%$ vs $1,5 \%, p>0,9)$, lo que se mantuvo al comparar sólo la recidiva de 
hernias unilaterales $(2 \%$ vs $4,1 \%, p=0,9)$. La Tabla 4 resume los resultados por grupo.

Un total de 128 pacientes tienen 1 año o más de seguimiento (65\% de la serie), lo que corresponde a 177 hernias operadas. Siete pacientes $(5,4 \%) 2 \mathrm{mu}-$ jeres y 5 cinco hombres, todos operados de hernia inguinal unilateral, presentaron dolor crónico.

Tabla 3. Características preoperatorias de los pacientes sometidos a hernioplastía laparoscópica por vía TAPP

\begin{tabular}{|lc|}
\hline Variable & $\mathbf{n}=\mathbf{1 9 7}$ \\
Edad, promedio \pm DS (años) & $52 \pm 15,4$ \\
Sexo, n (\%) & \\
M & $152(77,2)$ \\
F & $45(22,8)$ \\
ASA (\%) & \\
I & $114(57,9)$ \\
II & $80(40,6)$ \\
III & $3(1,5)$ \\
Lateralidad herniaria (\%) & \\
Derecha & $72(36,5)$ \\
Izquierda & $51(25,9)$ \\
Bilateral & $74(37,6)$ \\
Tiempo evolución, mediana (RIC) (meses) & $6(3-12)$ \\
Método diagnóstico (\%) & \\
Unilaterales & \\
$\quad$ Clínica & $115(93,5)$ \\
Ecografía & $8(6,5)$ \\
Bilaterales & \\
Clínica & $48(64,9)$ \\
Ecografía & $9(12,2)$ \\
Clínica-Ecografía & $13(17,5)$ \\
Clínica-Intraoperatorio & $4(5,4)$ \\
\hline
\end{tabular}

DS: desviación estándar, RIC: rango intercuartil.

\section{Discusión}

Existen varios estudios aleatorizados que comparan las técnicas laparoscópicas versus las técnicas abiertas en la reparación de hernias inguinales unilaterales. Un ensayo clínico del Reino Unido, que randomizó 300 pacientes a TEP o Lichtenstein con un seguimiento a 5 años del $48 \%$, demostró que no hubo diferencias en los grupos en cuanto a porcentaje de recidiva $(2 \%)$ ni dolor crónico ${ }^{15}$.

El meta-análisis de Cochrane del año 2003, revisó 41 ensayos clínicos que comparaban la hernioplastía abierta versus la laparoscópica con un total de 7.161 pacientes. Se encontró un mayor tiempo operatorio en el grupo laparoscópico, y un mayor porcentaje de complicaciones. Sin embargo, la reinserción laboral fue más temprana, hubo menos dolor crónico y no se encontraron diferencias significativas en cuanto al número de recidivas ${ }^{16}$.

En la presente serie, hubo un total de 5 recidivas en 271 hernioplastías laparoscópicas, lo que equivale al $1,85 \%$, cifra acorde con los estándares internacionales para la hernioplastía abierta con técnica de Lichtenstein, que en los mejores centros tiene una recidiva que varía desde el 0,5 al $2 \%{ }^{17,18}$.

A pesar de que en esta serie, se excluyeron los enfermos con hernia recidivada, parece existir suficientes argumentos en la literatura para plantear el abordaje laparoscópico como la mejor alternativa. Un estudio randomizado, que duró diez años, reclutó a 235 pacientes con recidiva herniaria luego de una cirugía con técnica de Lichtenstein y demostró menos complicaciones $(4,4 \%$ vs $12,2 \%)$ y menos re-recurrencia $(2,2 \%$ vs $5,7 \%)$ con el abordaje laparoscópico (TEP o TAPP) ${ }^{19}$.

En relación a las hernias inguinales bilaterales, Wauschkuhn y colaboradores, compararon 2.880 pacientes con hernia inguinal bilateral reparadas con técnica TAPP en un tiempo, con 7.240 pacientes con hernia unilateral reparada con la misma técnica, sin

Tabla 4. Resultados por grupo de la hernioplastía inguinal con técnica TAPP

\begin{tabular}{|c|c|c|c|c|c|}
\hline & $\begin{array}{c}\mathbf{n} \\
\text { pacientes }\end{array}$ & $\begin{array}{l}\text { T. operatorio } \\
\text { min. mediana }\end{array}$ & $\begin{array}{c}\text { Edad } \\
\text { Años }( \pm \text { DS })\end{array}$ & $\begin{array}{c}\text { Número de } \\
\text { complicaciones (\%) }\end{array}$ & $\begin{array}{c}\text { Recidiva } \\
\text { n }(\%)\end{array}$ \\
\hline H. Unilateral & 99 & 60 & $51 \pm 15$ & $15 \quad(15,2)^{*}$ & $2(2,02)$ \\
\hline H. Bilateral & 53 & 90 & $50 \pm 15$ & $6 \quad(11,3)^{* *}$ & $2(1,88)$ \\
\hline M. Unilateral & 24 & 45 & $49 \pm 14$ & $1 \quad(4,2)^{* * * *}$ & $1 \quad(4,16)$ \\
\hline M. Bilateral & 21 & 60 & $59 \pm 16$ & 0 & $0 \quad(0)$ \\
\hline Total & 197 & 60 & $52 \pm 15$ & $(11,2)$ & $5 \quad(1,85)$ \\
\hline
\end{tabular}

$\mathrm{H}$ : hombre, M: mujer, $\mathrm{N}^{\circ}$ : número, T: tiempo, min.: minutos, n: número de hernias, DS: desviación estándar. *Hematoma $(8$ pacientes), seroma (3 pacientes), retención urinaria (2 pacientes), epiplocele (1 paciente), infarto agudo al miocardio (1 paciente). ${ }^{* * \text { Seroma }}(3$ pacientes $)$, hidrocele ( 2 pacientes), hematoma (1 paciente). $* * *$ Seroma. 
encontrar diferencias en términos de dolor crónico, reinserción laboral ni recidiva ${ }^{20}$. En nuestra serie los 74 pacientes con hernia inguinal bilateral se resolvieron en un tiempo quirúrgico, sin diferencias estadísticamente significativas en términos de recidiva al compararlas con las unilaterales.

Un escenario especial lo representan las mujeres, pues algunos autores sugieren la vía laparoscópica como la de elección. Esto sustentado en la alta incidencia de hernia femoral sincrónica que alcanza hasta el $40 \%{ }^{21}$ en las mujeres. En nuestra serie el sexo femenino no fue un elemento que gravitara a favor de la elección de la técnica laparoscópica y como se muestra en los resultados no hubo diferencias estadísticamente significativas al compararlas con los hombres.

En relación al tipo de prótesis a utilizar no existe evidencia que sustente el uso de un tipo de malla por sobre otro para la técnica TAPP. Sin embargo, según las guías clínicas del año 2011, se recomienda el uso de mallas de monofilamento, cuyo poro sea de al menos 1,5 mm (evidencia tipo V). Sin embargo, lo único demostrado, es que las mallas de bajo peso presentan ventajas en relación a la convalecencia del paciente durante las dos primeras semanas (evidencia IA), al compararlas con las mallas de alto peso ${ }^{22}$.

Finalmente, en este estudio los resultados de la técnica TAPP para la reparación de hernia inguinal mostraron ser similares a los publicados en la literatura.

\section{Referencias}

1. Llanos O. Historia de la cirugía de la hernia inguinal. Rev Chil Cir. 2004;56:404-9.

2. Lau WY. History of treatment of groin hernia. World J Surg. 2002;26:748-59.

3. Haeger K. The illustrated history of surgery. Harold Starke Publishers Ltd. London, United Kingdom, 2000.

4. Rutkow I. Surgery. Ann illustrated history. St Louis, USA: Mosby 1993.

5. Amato B, Moja L, Panico S, Persico G, Rispoli C, Rocco N, et al. Shouldice technique versus other open techniques for inguinal hernia repair. Cochrane Database Syst Rev. 2009 Oct 7;CD001543.

6. Read RC. Crucial steps in the evolution of the preperitoneal approaches to the groin: an historical review Hernia. 2011;15:1-5.

7. Lichtenstein IL, Shulman AG, Amid PK, Montloor MM. The tension-free hernioplasty. Am J Surg. 1989;157:188-93.

8. Amid PK, Shulman AG, Lishtenstein IL. The Lichtenstin herniotomy procedure. Chirug. 1994;64:54-8.
9. Ger R. The management of certain abdominal herniae by intra-abdominal closure of the neck of the sac. Preliminary communication. Ann R Coll Surg Engl. 1982;64:342-4.

10. Arregui ME, Davis CJ, Yucel O, Nagan RF. Laparoscopic mesh repair of inguinal hernia using a preperitoneal approach: a preliminary report. Surg Laparosc Endosc. 1992;2:53-8.

11. Nicholson T, Tiruchelvan V. Comparision of laparoscopic vs open modifed Shouldice technique in inguinal hernia repair. JSLS 1999;3:39-43.

12. Liem M, van der Graaf Y, van Steensel CJ, Boelhouwer RU, Clevers GJ, Meijer WS. Comparison of Conventional Anterior Surgery and Laparoscopic Surgery for Inguinal-Hernia Repair. N Engl J Med. 1997;336:15417.

13. Nyhus LM. Individualization of hernia repair: a new era. Surgery 1993;114:102.

14. Kaafarani HM, Hur K, Campasano M, Reda DJ, Itani KM. Classification and valuation of postoperative complications in a randomized trial of open versus laparoscopic ventral herniorrhaphy. Hernia 2010;14:231-5.

15. Wright D, Paterson C, Scott N, Hair A, O'Dwyer PJ. Five-year follow-up of patients undergoing laparoscopic or open groin hernia repair: a randomized controlled trial. Ann Surg. 2002;235:333-7.

16. McCormack K, Scott NW, Go PM, Ross S, Grant AM. EU Hernia Trialists Collaboration. Laparoscopic techniques versus open techniques for inguinal hernia repair. Cochrane Database Syst Rev. 2003;CD001785. Review.

17. Beltrán M, Burgos C, Paredes M, Martínez H, Larenas $\mathrm{R}$, Tapia T, et al. Resultados y seguimiento alejado de la hernioplastía de Lichtenstein: Aplicación de un instrumento cualitativo y cuantitativo de medición. Rev Chil Cir. 2005;57:320-9.

18. Uzzaman MM, Ratnasingham K, Ashraf N. Metaanalysis of randomized controlled trials comparing lightweight and heavyweight mesh for Lichtenstein inguinal hernia repair. Hernia 2012;16:505-18.

19. Itani KM, Fitzgibbons R Jr, Awad SS, Duh QY, Ferzli GS. Management of recurrent inguinal hernias. J Am Coll Surg. 2009;209:653-8.

20. Wauschkuhn CA, Schwarz J, Boekeler U, Bittner R. Laparoscopic inguinal hernia repair: gold standard in bilateral hernia repair? Results of more than 2800 patients in comparison to literature. Surg Endosc. 2010;24:302630 .

21. Carter J, Duh QY. Laparoscopic repair of inguinal hernias. World J Surg. 2011;35:1519-25.

22. Bittner R, Arregui ME, Bisgaard T, Dudai M, Ferzli GS, Fitzgibbons RJ, et al. Guidelines for laparoscopic (TAPP) and endoscopic (TEP) treatment of inguinal hernia [International Endohernia Society (IEHS)]. Surg Endosc. 2011;25:2773-843. 